\title{
Fraunhofer IKTS baut Entwicklung für Transparentkeramik aus
}

\begin{abstract}
Das Fraunhofer-Institut für Keramische Technologien und Systeme IKTS übernahm zum 1. April 2021 die Transparentkeramik-Sparte des Unternehmens Ceramtec-ETEC. Das Institut weitet damit seine bestehenden Kompetenzen in der Entwicklung von Transparentkeramiken aus. Die hinzugewonnene technische Infrastruktur ermöglicht den Aufbau eines Forschungs- und Entwicklungszentrums für transparente Keramik am IKTS-Standort Hermsdorf.
\end{abstract}

Die Technologiekette zur Transparentkeramik des Unternehmens Ceramtec-ETEC wird in den nächsten Monaten am thüringischen Standort des IKTS in Hermsdorf integriert. Sie ergänzt die dort bereits bestehenden Anlagen und ermöglicht die Herstellung hochreiner, transparenter Keramik für optische Systeme, Medizintechnik und ballistischen Schutz (Bild 1). Mit der finanziellen Unterstützung des Freistaats Thüringen in Höhe von 2,5 Mio. Euro entsteht am Standort Hermsdorf des Fraunhofer IKTS ein Forschungs- und Entwicklungszentrum, das mit modernsten Technologien dazu beitragen wird, die bisherigen Einsatzszenarien von Transparentkeramiken deutlich zu erweitern. „Transparentkeramiken decken ein weites

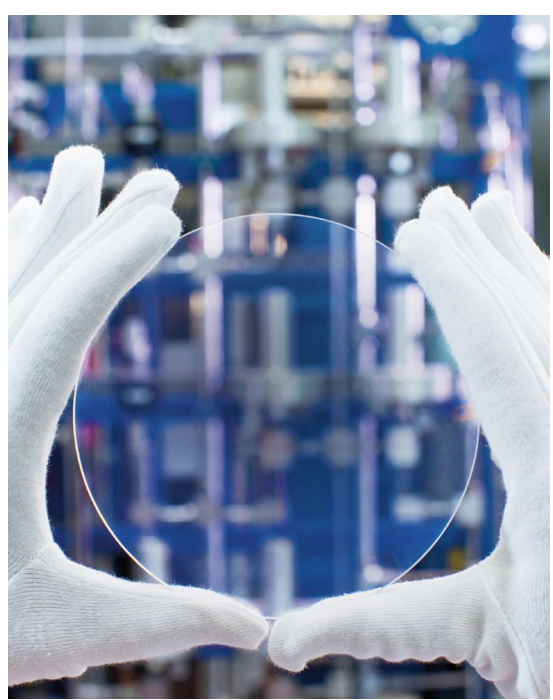

Bild 1 Das Fraunhofer IKTS weitet seine Aktivitäten im Bereich der Forschung und Entwicklung von Transparentkeramik aus. (@ Fraunhofer IKTS)
Einsatzspektrum ab, das von der Optik über die Medizintechnik bis hin zur Industrie 4.0 reicht", sagte Thüringens Wirtschaftsminister Wolfgang Tiefensee. Beispiele seien kratzfeste Displays und Schaltflächen, Schutzabdeckungen für optische und Sensorsysteme im Außeneinsatz, Sicherheitsglas und großformatige Scannerscheiben (Bild 2). „Das alles sind Anwendungen und Technologiefelder, die künftig eine hohe Nachfrage nach Transparentkeramiken generieren werden. Als Land unterstützen wir es deshalb, dass das Fraunhofer IKTS in Hermsdorf seine Kompetenzen in diesem wichtigen und schnell wachsenden Markt verstärkt.“

In Hermsdorf wird eine komplette keramische Technologiekette, bestehend aus Anlagen zur Konditionierung von hochreinen Pulvern, Formgebung, Laserbearbeitung und Ultrapräzisions-Finish-Bearbeitung aufgebaut, die ein Bindeglied zwischen Labormaßstab und industrieller Fertigung darstellt. Die Technologie der Transparentkeramik in Hermsdorf wird damit einen Performancesprung hinsichtlich Bauteilgröße, Reinheitsgrad und innovativer Fertigungsmethoden erreichen. Als wichtige Grundlage für zukünftige Projekte dient dabei die Förderung durch den Freistaat. Sie ermöglicht am Standort einen echten Meilenstein beim Ausbau der Region Ostthüringen zum europaweit führenden Zentrum für Hochleistungskeramik.

\section{Zahlreiche Einsatzszenarien für Transparentkeramiken}

Die besonderen optischen und mechanischen Eigenschaften von transparenter

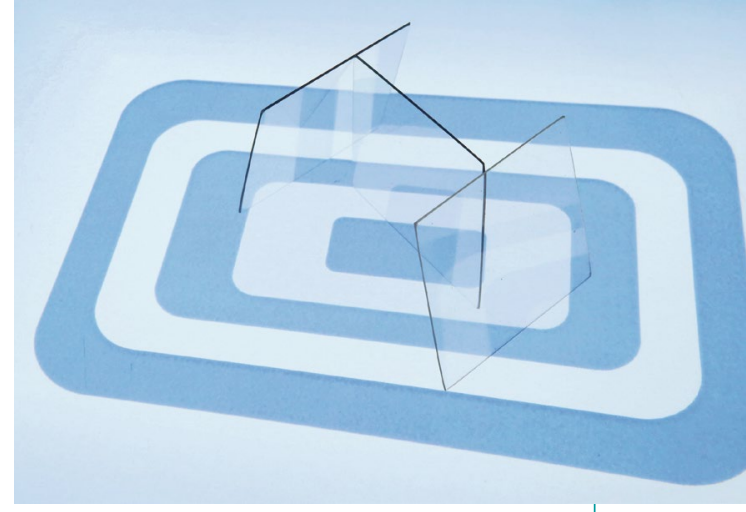

Bild 2 Transparente Keramik (hier: Spinellkeramik) kann zum Beispiel für hochbeanspruchte Displays von mobilen Endgeräten eingesetzt werden. (৫) Fraunhofer IKTS)

Keramik eröffnen ein breites Anwendungsspektrum. Das Material zeichnet sich neben einer hohen Transparenz durch eine hohe Festigkeit und enorme Härte aus - es ist etwa drei bis viermal so hart wie konventionelles Glas. Transparentkeramik kann damit unter anderem in Optik- und Sensorsystemen (Kameraoptiken für Fahrassistenzsysteme, Lidar-Sensoren für autonomes Fahren), im Zivilschutz, in der Medizintechnik (Endoskope für Human- und Veterinärdiagnostik) oder in Displays und Schaltflächen unter harschen Umgebungsbedingungen eingesetzt werden.

Kontakt:

Fraunhofer-Institut für Keramische Technologien und Systeme IKTS,

07629 Hermsdorf, www.ikts.fraunhofer.de 